\title{
INDÚSTRIA 4.0: REVOLUÇÃO E IMPACTO NO MUNDO MODERNO
}

\author{
Adão Maciel Monteiro Chmielewski \\ Depto. de Engenharia Elétrica - Universidade Santa Úrsula (USU), Rio de Janeiro, RJ, Brasil \\ adao.maciel@usu.edu.br \\ Antônio José Dias da Silva \\ Depto. de Engenharia Elétrica - Universidade Santa Úrsula (USU), Rio de Janeiro, RJ, Brasil \\ antonio.dias@usu.edu.br \\ José Roberto Emiliano Leite \\ Faculdade de Tecnologia da Universidade de Campinas - FT/UNICAMP, São Paulo, Brasil \\ joserobertoemilianoleite@gmail.com
}

\begin{abstract}
RESUMO
Um grande desafio tecnológico começa a se tornar realidade no mundo atual, pois já está causando um grande impacto em toda a humanidade. Esta revolução é conhecida como a quarta revolução industrial ou indústria 4.0, a qual teremos bilhões de pessoas conectadas por dispositivos móveis, com poder de processamento, recursos de armazenamento e acesso ao conhecimento sem limites. Essa nova onda tecnológica incluirá diversas áreas: inteligência artificial (IA), robótica avançada, internet das coisas (IoT, na sigla em inglês), veículos autônomos, big data, armazenamento em nuvem, realidade aumentada, sistemas cyber físicos, segurança digital, impressão 3D, entre outras. O objetivo desse trabalho é mostrar historicamente como as revoluções industriais vieram evoluindo desde a 1a até a 4 a revolução ou Indústria 4.0. A partir daí, definir seu conceito, explicar cada tecnologia que a envolve, dar exemplo de aplicações e analisar os impactos em vários setores. Para isso foi realizado um levantamento bibliográfico de artigos publicados no Portal de Periódicos da Capes, nos últimos dez anos. Por consequência observou-se que essa nova tecnologia vem sendo cada vez mais incorporada pelas grandes empresas, como uma nova tendência a ser explorada, possibilitando mais segurança e produtividade.
\end{abstract}

Palavras-chave: Industria 4.0, Tecnologias 4.0, IoT, Aplicações loT, Inteligência Artificial.

\section{INDUSTRY 4.0: REVOLUTION AND IMPACT ON THE MODERN WORLD}

\begin{abstract}
A major technological challenge is beginning to become a reality in today's world, as it is already having a great impact on all of humanity. This revolution is known as the fourth industrial revolution or industry 4.0, which will have billions of people connected by mobile devices, with unlimited processing power, storage resources and access to knowledge. This new wave of technology will include several areas: artificial intelligence (Al), advanced robotics, internet of things (IOT), autonomous vehicles, big data, cloud storage, augmented reality, cyber-physical systems, digital security, printing 3D, among others. The objective of this work is to show historically how industrial revolutions have evolved from the 1st to the 4th revolution or Industry 4.0. From there, define its concept, explain each technology that involves it, give examples of applications and analyze the impacts in various sectors. For this, a bibliographic survey of articles published in the Capes Journal Portal in the last ten years was carried out. Consequently, it was observed that this new technology has been increasingly incorporated by large companies, as a new trend to be explored, enabling more security and productivity.
\end{abstract}

Keywords: Industry 4.0, Technologies 4.0, loT, loT Applications, Artificial Intelligence.

\begin{tabular}{l|l|l|l|l|l|r} 
Projectus | & Rio de Janeiro & V.5 & N.1 & P. 1-12 & 2020
\end{tabular}




\section{INTRODUÇÃO}

No início do século 18 na Grã-Bretanha, iniciou-se a primeira revolução industrial com o aparecimento da máquina a vapor, tendo sido largamente utilizada na produção têxtil. As manufaturas são substituídas, pelas indústrias. Já a segunda Revolução Industrial, iniciou-se na segunda metade do século 19, com a eletricidade tendo papel fundamental nas mudanças, sendo as residências beneficiadas, além de máquinas e motores apresentarem dimensões cada vez mais reduzidas. A Terceira Revolução Industrial começou em meados do século 20 , graças a invenção do transistor que impulsionou a eletrônica com circuitos e componentes pequenos, mais velozes e estáveis, sendo responsável pela modernização da indústria e passando a ser dominada por Controladores Lógicos Programáveis (CLPs). Surge então a introdução da informática nos processos de fabricação. Aparece o computador no processo fabril para supervisão e controle, além dos primeiros sistemas de comunicação (BRANCO, 2020).

Atualmente, temos a quarta Revolução Industrial, ou Indústria 4.0 que começou a ser difundida na segunda década do século 21 , com a revolução digital, isto é, a internet muito mais móvel e global e a integração de sensores menores e mais poderosos, machine learning e inteligência artificial amparados por requisitos de comunicação e intercomunicação de forma maciça em rede, estabelecidos globalmente. Como consequência, surge um novo paradigma na organização do trabalho e lazer questionando a visão tradicional e gerando graus de complexidade e interconexão entre os setores (SCHWAB, 2019).

Este tema torna o estudo interessante em que se procurará detalhar melhor o conceito e definição da Industria 4.0, com explicações de suas tecnologias, mostrando de fato que é uma revolução sem precedentes, com exemplos de aplicações, e seus impactos positivos em algumas áreas do mundo moderno.

\section{DESENVOLVIMENTO: CONCEITOS E DEFINIÇÃO EM ABERTO DA INDUSTRIA 4.0}

\subsection{Conceitos}

Estamos no início de uma revolução que alterará profundamente a maneira como vivemos, trabalhamos e nos relacionamos. Em sua escala, escopo e complexidade, a quarta revolução industrial ou Industria 4.0, é algo diferente de tudo aquilo que já foi experimentado pela 
humanidade. É uma transição em direção a novos sistemas que foram construídos sobre a infraestrutura da revolução digital (SCHWAB, 2019).

\subsection{Definição}

A Indústria 4.0 possui uma capacidade maior de integração e conectividade entre pessoas, máquinas, e fabricas, devido ao surgimento de materiais avançados, que permitem 0 desenvolvimento de novos sensores, tecnologia de processamento mais rápido, redes de produção avançadas, de dispositivos de fabricação controlados por computadores e em alguns casos já automatizados com robôs autônomos, permitindo uma interação entre o real e o virtual, através das tecnologias tais como: inteligência artificial (IA), robótica avançada, internet das coisas (loT), veículos autônomos, big data, armazenamento em nuvem, realidade aumentada, sistemas cyber físicos, segurança digital, impressão 3D. A definição de Industria 4.0 encontra-se em aberto, pois a mesma está surgindo e se expandindo, tornando o futuro imprevisível.

\section{TIPOS DE TECNOLOGIA 4.0}

A quarta revolução industrial se caracteriza por um conjunto de tecnologias que permitem a fusão do mundo físico, digital e biológico (FIGURA 1).

Figura 1: Tecnologias da Indústria 4.0.

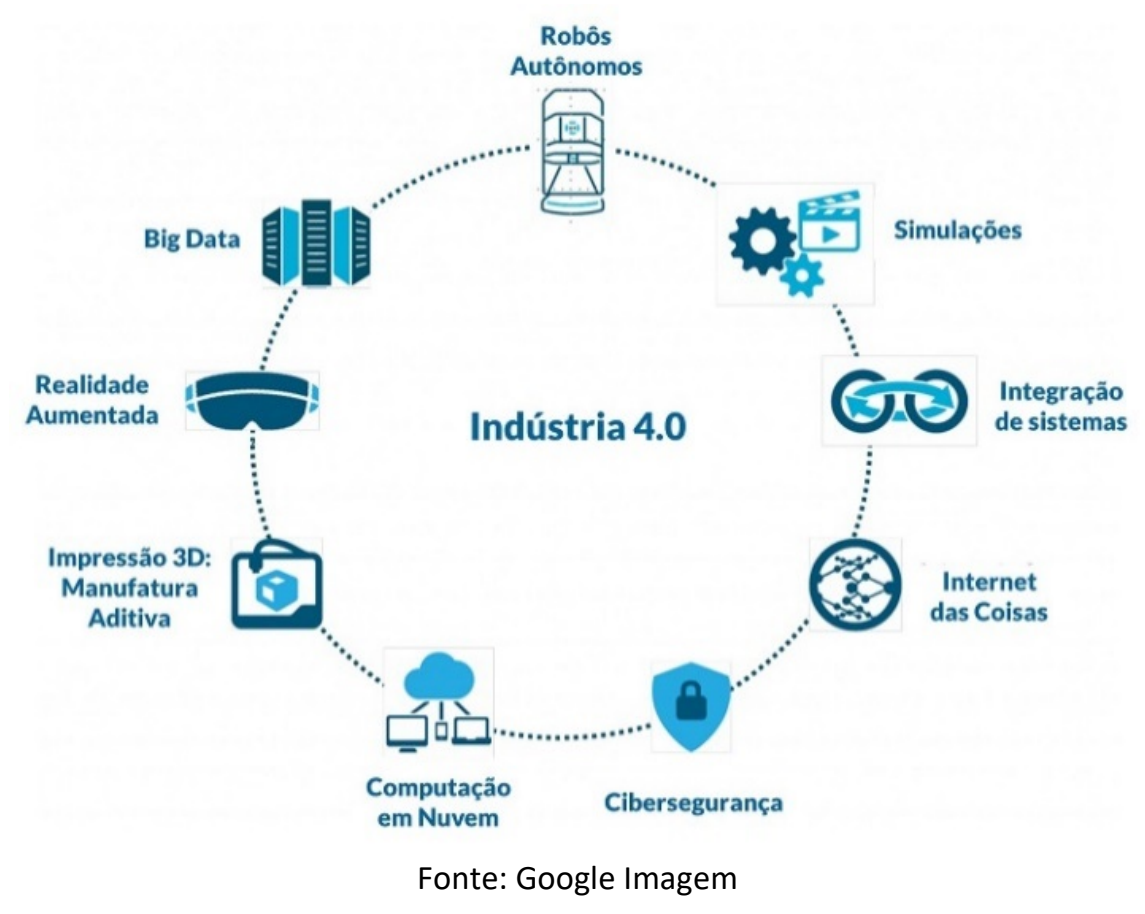




\subsection{Robôs Autônomos ou Avançados}

Estes realizam atividades e cumprem objetivos sem a navegação ou intervenção humana. $\mathrm{Na}$ Indústria 4.0. Possuem também a capacidade de "aprender sozinho" e reformular sua estratégia de acordo com o ambiente em que estão. Além disso, eles conseguem interagir com outras máquinas e realizar tarefas de forma mais rápida e segura, resultando na redução de custos.

\subsection{Big Data}

O Big Data deve ser capaz de lidar com um grande volume de dados e é utilizado para definir um grande conjunto de ferramentas de TI que permitem a captura, análise e a catalogação de dados em tempo real. E está baseado em 5 princípios: volume, variedade, velocidade, veracidade e valor.

\subsection{Computação ou Armazenamento em Nuvem}

A nuvem não é um lugar, mas um método de gerenciar os recursos de TI que substitui máquinas locais e data centers privados por uma infraestrutura virtual. Nesse modelo, os usuários acessam recursos virtuais de computação, rede e armazenamento disponibilizados online por um provedor remoto. Esses recursos podem ser provisionados de forma instantânea.

\subsection{Realidade Aumentada}

É uma tecnologia que permite que o mundo virtual seja misturado ao real, possibilitando maior interação e abrindo uma nova dimensão na maneira como nós executamos tarefas, ou mesmo as que nós incumbimos às máquinas.

\subsection{Impressão 3D}

Também conhecida como prototipagem rápida, é uma forma de tecnologia de manufatura aditiva onde um modelo tridimensional_é criado por sucessivas camadas de material. Por não 
necessitar do uso de moldes e permitir produzir formas que não são viáveis em outros métodos de produção.

\subsection{Cybersegurança}

Cybersegurança é a prática que protege computadores e servidores, dispositivos móveis, sistemas eletrônicos, redes e dados contra-ataques maliciosos. Também é chamada de segurança da tecnologia da informação ou segurança de informações eletrônicas.

\subsection{Internet das Coisas}

É uma extensão da INTERNET atual que possibilita que objetos do dia-a-dia, quaisquer que sejam, mas que tenham capacidade computacional e de comunicação, se conectem à Internet, possibilitando em primeiro lugar, controlar remotamente os objetos e, em segundo lugar, que os próprios objetos sejam usados como provedores de serviços.

\subsection{Integração de Sistemas}

O objetivo é conectar as diferentes áreas de uma indústria, a fim de extrair dados e informações que serão usadas para realizar melhorias contínuas em todo o processo produtivo e nas áreas de suporte relacionadas. A integração pode ser horizontal em que se refere a sistemas de TI e fluxos na cadeia de fornecimento/valor, incluindo os vários processos que passam por ela ou pode ser vertical, em que permite que todos os níveis da fábrica estejam conectados, do chão de fábrica até os executivos.

\subsection{Simulações}

Simulação é a reprodução virtual de ambientes e processos de desenvolvimento complexos, e manufatura nas fábricas. É uma prática que possibilita a reprodução digital e fiel do funcionamento das plantas industriais, abrangendo equipamentos, funções operacionais e de funcionários. Com o objetivo de melhor monitorá-las, de modo a prever dificuldades e aprimorar a produção. 


\section{APLICAÇÕES DA INTERNET DAS COISAS (IoT)}

A Internet consagrou-se como sendo uma "Rede de Redes" e uma ferramenta de comunicação globalizada, inicialmente possibilitando a ligação entre pessoas e entre pessoas e computadores (máquinas). Foi criada nos anos 60 com o objetivo de interligar os ambientes acadêmicos e de pesquisas; com seu crescimento, a Internet foi expandida para a área comercial, aumentando ainda mais o seu uso com o surgimento da forma de busca WEB (WWW) e das redes sociais. Atualmente, é impossível imaginarmos nossa vida sem o uso da Internet, das redes sociais e comerciais (EMILIANO 1,2019$)$.

O barateamento e a diminuição do tamanho dos componentes de acesso à Internet possibilitaram que equipamentos e aparelhos mais simples de nosso cotidiano pudessem também acessar a rede global (TV, geladeira, fogão, ar-condicionado, alarmes residenciais, lâmpadas, automóveis, aparelhos hospitalares); surgiu então a Internet das Coisas (IoT). Essa nova forma de utilização da Internet visa interligar aparelhos de uso cotidiano, executando assim uma comunicação entre coisas/objetos/aparelhos, possibilitando uma maior automatização do nosso cotidiano, por meio do uso do acesso celular. Possibilita também o incremento de inteligência em diversos setores da economia: Smart Grid (Setor Elétrico), Cidade e Casas Inteligentes, Indústria, Saúde, Lazer e Automatização Comercial (atacado e varejo), entre outros. A inteligência e a automação são decorrentes dos acréscimos de processamento, memória e comunicação nos objetos envolvidos.

\subsection{Arquiteturas, Modelos, Protocolos e Tecnologias selecionadas pela loT}

A Arquitetura IoT foi padronizada internacionalmente em [IDA], sendo que o estudo de

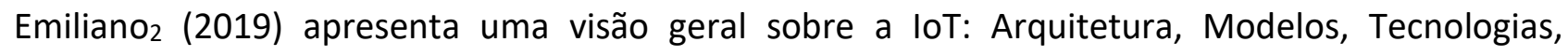
Protocolos e Aplicações.

A Arquitetura loT escolheu Tecnologias Abertas, Simples, Baratas e Consagradas como Ethernet, WiFi, Ad-Hoc, ZIGBEE, Bluetooth, RFID (Radio-Frequency Identification), WSS (Wireless

Signal Solutions) e Rede de Sensores. É claro que soluções cabeadas como Ethernet/Wired também podem ser usadas. A Arquitetura loT selecionou outras Arquiteturas consagradas como TCP/IP e SOA (Service Oriented Architecture). A Arquiteura IOT separa a Comunicação nas seguintes Camadas: Conectividade de Sensores e Redes; Gateways e Redes; Serviços de Gerenciamento de Rede e Segurança da Informação, e Aplicações. 
A loT escolheu também aplicações de utilização direta na sociedade e na Economia Mundial, como por exemplo: Ambiental, Energia (Smart Grid), Transporte, Saúde, Varejo/Atacado, Cadeia de Produção, Acompanhamento de Pessoas (Healthcare) e Segurança (Smart Home, Building, City), entre outros. O Modelo de Referência loT conforme o entendimento de Telecomunicações do ITU-T, ISO e IEC, também segue o modelo camadas (Application, Service and Application Support, Network e Device). Como todos os modelos de referência da ITU-T, possui 2 colunas referentes a Gerenciamento de Rede e Segurança, colunas essas que tem funcionalidades espalhadas em todas as camadas do modelo.

O Modelo de Comunicação loT foi baseado no Modelo OSI(Open System Interconnection) da ISO. O foco principal é a informação trocada ("Data") entre o objeto e a aplicação. A maior parte dos protocolos utilizados na IoT e na WEB foram aproveitados da Arquitetura TCP/IP. A IoT selecionou diversas tecnologias padronizadas e já utilizadas internacionalmente.

Assim sendo, muito dos novos ambientes loT são parecidos com os atuais existentes na INTERNET Residencial e Empresarial. A maioria das tecnologias é por rádio e sem fio (Radio Access Technologies), ou seja, utilizando os padrões IEEE 802.11, IEEE 802.15 e IEEE 802.16, por questões de flexibilidade e alcance.

\subsection{Aplicações selecionadas pela loT}

A IoT selecionou diversas aplicações em diversos Setores da Economia Mundial: Cliente e Residência (Conforto e Conveniência); Prédios (Comercial e Institucional); Indústria (automação); Cuidado com a Saúde; Energia, água, gás e telecom; Mercado (Atacado e Varejo); Transporte; Segurança Pública; Tecnologia da Informação e Redes (TICS).

Smart Home visa oferecer automação residencial usando o Smartphone ou Tablet para acesso remoto, como por exemplo: acender e apagar luzes, saber o tempo de vida das lâmpadas, abrir e fechar portas e portões, ligar e desligar TV/Ar-Condicionado, receber a lista de mantimentos faltantes na geladeira, receber informações via SMARTTV e Videos, etc.

Smart Building visa a automatização em um edifício, tornando 'green" em termos de Energia Elétrica, Água, Aquecimento Solar, Sistemas de Baterias, etc. Equipamentos de alto consumo (Ar-Condicionado e Iluminação) poderão ser ajustados dinamicamente em função da temperatura/umidade/claridade interna e externa do prédio. Sistemas de Baterias poderão ser supervisionados visando a antecipação de troca em caso de futura falha. 
Smart Cities trata de troca de informações entre carros, celulares, prédios e semáforos, visando melhorar o trânsito e fluxo de carros em uma cidade. A Smart City abrange as seguintes áreas: Saúde das Estruturas de Prédios e Pontes; Monitoramento de Lixo; Monitoramento da qualidade do ar; Monitoramento de ruído; Monitoramento do Congestionamento de Tráfego; Consumo de energia na cidade; Estacionamentos Inteligentes; Semáforos Inteligentes; Automação e Salubridade em prédios públicos. O ITS (Intelligent Transportantion System) é parte funcional da Smart City em estradas.

Smart Grid (Energy e Utilities) cuida da Automatização do Setor Elétrico visando colocar nas residências leitores inteligentes (Smart Meters) que passam as informações de consumo diretamente para as empresas fornecedoras, as quais utilizam a própria rede para gerenciar seus equipamentos e falhas. Utiliza uma rede específica do setor elétrico com tecnologia PLC (Power Line Communication), por questões de confiabilidade e tempo de resposta. Seus principais componentes são: Geração; Transmissão; Automação da Distribuição; Carros Elétricos e Gerenciamento da Energia Elétrica na Residência).

Healthcare (Assistência Médica Remota) possibilita o monitoramento e acompanhamento remoto de pacientes em suas casas, através da coleta de informações de sensores (no paciente) de temperatura/pressão arterial/ batimentos cardíacos/nível de glicose/nível de oxigenação, sensores ambientais de presença/ temperatura/pressão/movimento, RFIDs de crachás de paciente, enfermeira, cuidadora, funcionários, remédios, equipamentos, etc, informações essas que são enviadas para a nuvem e são acessadas pelos médicos e hospitais. O médico poderá acessá-las através de seu Smartphone e solicitar que o paciente seja deslocado para o hospital em caso de valores perigosos nos seus sinais vitais. Essa aplicação possibilita um novo modelo de negócio para Planos de Saúde, em que o paciente só vai ao Hospital em situações graves.

Smart Manufacturing (Industry) é responsável pelo controle e automação de processos fabris reais (Indústria 4.0) sobre Dispositivos Indústriais (Tornos, Prensas, Controladores Numéricos, Controladores de Lógica Programáveis (PLCS), Robôs, etc), requerendo tempos de reação curtos (Real Time), com pouco atraso e jitter, e pouca quantidade de dados produzida. Adaptadores, Bridges e Gateways são necessários devido ao grande número de Ilhas (Aplicações) existentes com protocolos proprietários.

Smart Farming automatiza a fazenda, através da sua interconexão com o mundo global. Possibilita a interconexão de tratores, colheitadeiras, drones, sede da fazenda, cooperativas, entidades da agricultura e aviões pulverizadores. Os funcionários e os animais são identificados através de etiquetas RFIDs. É necessário a instalação de uma Estação Rádio Base na fazenda, 
permitindo a interconexão dessas diversas partes que permite construir uma fazenda ONLINE. Esta aplicação é de grande importância para o Brasil por ser um país fortemente agrícola.

\section{APLICAÇÃO DE INTELIGÊNCIA ARTIFICIAL - UTILIZAÇÃO DE REDES NEURAIS NO RECONHECIMENTO DE PADRÕES}

Segundo Figueiredo (2019), o objetivo do projeto foi desenvolver um software que é capaz de identificar objetos através de algoritmos de reconhecimento de padrões utilizando Redes Neurais. Para isso é necessário fazer a leitura da imagem de uma webcam, converter o bitmap RGB (vermelho, verde, azul) em escala de cinza, aplicar filtros para que fique apenas em preto e branco e finalmente realizar medições. Alguns dos parâmetros que foram medidos são área quadrada, centroide, forma, perímetro e a cor do objeto. Estes dados devem alimentar a rede neural a fim de que se faça o aprendizado de máquina. Após isso o programa está apto a fazer o reconhecimento de objetos e qualificá-los.

O software de reconhecimento de padrões foi desenvolvido conforme a Figura 2.

Figura 2: Esquemático do funcionamento do software de reconhecimento.

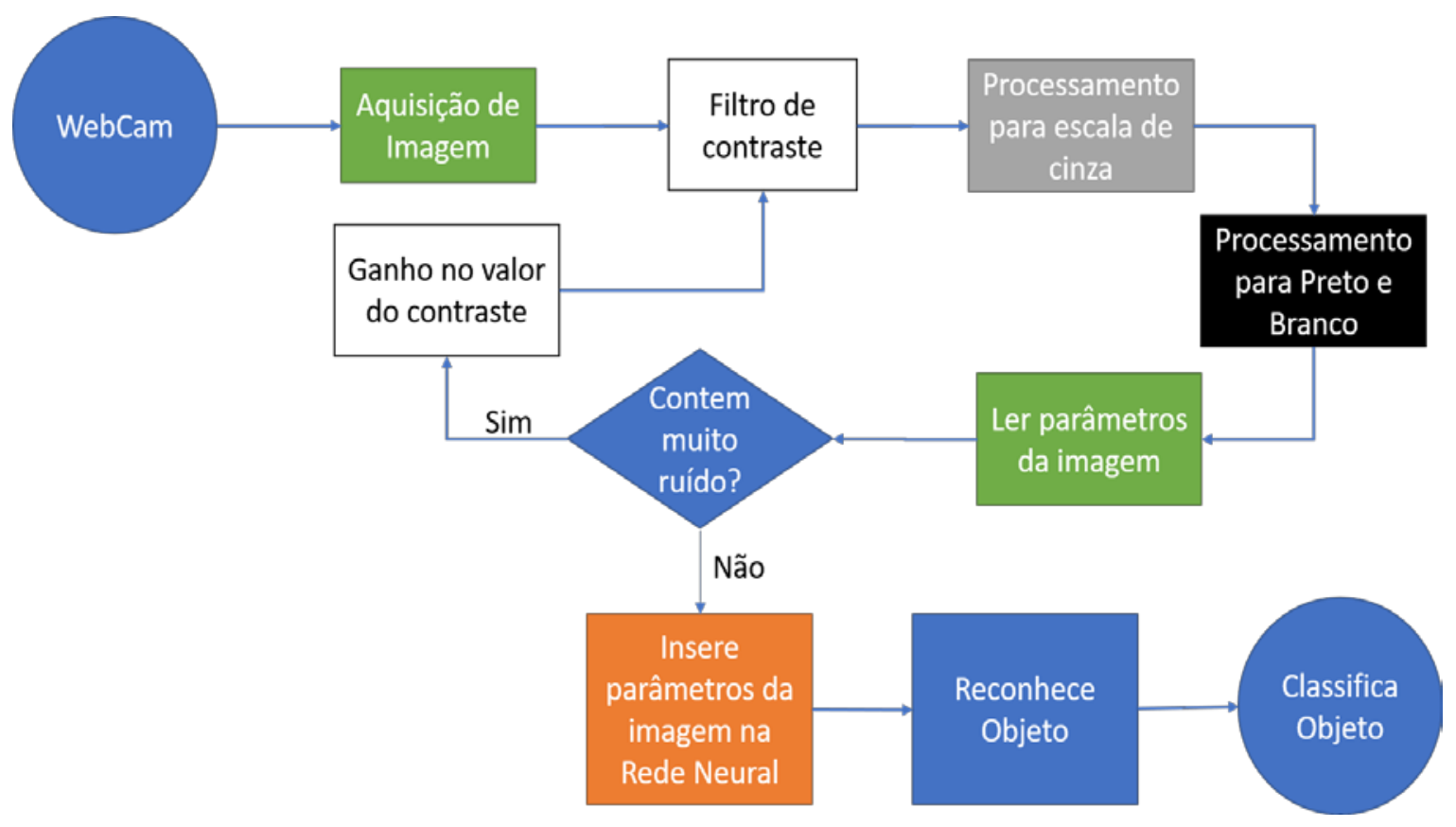

Fonte: Figueiredo, 2019

Aquisição de Imagem é a etapa de escolher uma biblioteca capaz de ler através das webcams atuais, com disponibilidade na tecnologia Microsoft DOTNET e principalmente fosse de livre distribuição. Portanto, foi escolhida a biblioteca da AFORGE.NET, que é uma estrutura C \# projetada para desenvolvedores e pesquisadores nas áreas de Visão Computacional e Inteligência 
Artificial - processamento de imagens, redes neurais, algoritmos genéticos, aprendizado de máquina, robótica. Com isso foi possível escolher o dispositivo que será feita a aquisição da imagem, escolher os frames para se fazer a análise (AFORGE, 2019).

\section{ALGUNS EXEMPLOS DE IMPACTOS NO MUNDO MODERNO}

\subsection{Indústria}

De grande impacto positivo, a Indústria 4.0 tem o objetivo de integrar todos os padrões de comunicação em ambiente industrial já estabelecido, dentro de uma proposta de comunicação, em que o protocolo IP passa a ser comum em todos os ambientes da produção, de forma a permitir a integração proposta. Ou seja, integração Máquina a Máquina (M2M), quando as informações passam a ser trocadas entre as máquinas, sem necessariamente haver uma comunicação com níveis. Quer dizer fábricas inteligentes, capazes de operar de maneira autônoma. Esses recursos descentralizam o controle dos processos e garantem inteligência para que as máquinas agendem manutenções, prevejam falhas e se adaptem às mudanças não planejadas.

Entre as características mais marcantes teremos a interoperabilidade: onde Pessoas, máquinas, dispositivos e sensores se comunicam entre si por meio da Internet das Coisas (IoT) e da Cloud Computing a troca de informações é constante e gera dados para a indústria ser, de fato, inteligente. Capacidade de operação em tempo real, onde dispositivos inteligentes e interconectados coletam e entregam dados interpretados em tempo real. Com isso, as tomadas de decisões são imediatas. Virtualização em Sensores de dados instalados por toda a fábrica, permite a criação de uma cópia virtual da indústria. Assim, é possível fazer monitoramento remoto de todos os processos, prever falhas e realizar manutenções preditivas, produtivas, a fim de aprimorar a operação. Orientação a serviços que está aliado ao conceito de Internet of Services (IoS) - Internet dos Serviços, softwares extraem dados e fazem análises de como os dispositivos estão funcionando e o que eles estão fazendo. Assim, é possível padronizar processos específicos e controlar atividades para garantir rentabilidade máxima (STEVAN, 2018).

\subsection{Volume de Dados}

A sociedade tornou-se hiper conectada, que participa ativamente do envio e recebimento de dados através da internet. As redes sociais, dados de Global System Positioning (GPS), 
mensagens de texto, envio de vídeos, registro de transações de compras, cliques em sites, sensores em dispositivos, entre outros, fazem parte de um grande banco de dados não estruturado. Segundo INTEL (2012), a civilização criou até 2003 cinco exabytes (exa $=10^{18}$ ) de informação e hoje esse volume é criado em apenas dois dias. Em 2012, o universo digital de dados cresceu 2,72 zettabytes ( $\left(\right.$ etta $\left.=10^{21}\right)$ ZB) e irá dobrar a cada dois anos, atingindo 8 ZB em 2015. Isso equivale ao acervo de 18 milhões de Bibliotecas do Congresso dos Estados Unidos. Então sem a tecnologia da Big data seria impossível processar massa tão gigantesca de dados e a sociedade não teria mais o controle dos dados.

\subsection{Biologia}

Especula-se por exemplo que o próximo passo é a biologia sintética. Pois a integração de diferentes tecnologias avançadas faz com que elas se desenvolvam mutuamente, a impressão em 3D será aliada à edição de genes para produzir tecidos vivos, reparação e regeneração dos tecidos num processo chamado bio impressão tridimensional. A técnica já foi utilizada para criar pele, osso, coração e tecido vascular, e está próximo de surgirem camadas impressas de células do fígado que serão usadas para gerar órgãos transplantáveis.

\section{CONSIDERAÇÕES FINAIS}

Na Indústria 4.0, a conexão digital com o avanço da inteligência do software está mudando profundamente a sociedade. Esta transformação devido a sua abrangência e a velocidade que ocorre, talvez seja diferente de toda a revolução industrial que a humanidade já passou. Pode-se afirmar que é uma mudança de paradigma que causa um novo modo de viver.

Além disso, essa tecnologia vem sendo cada vez mais incorporada pelas grandes empresas, como uma nova tendência a ser explorada, possibilitando mais segurança e produtividade.

Enfim, a previsão é de que a quarta revolução industrial gere grandes impactos no mercado de trabalho e nas ofertas de mão-de-obra. Espera-se que, aos poucos, os trabalhos manuais e repetitivos sejam substituídos por ferramentas e recursos automatizados, ou seja, robôs e máquinas. 


\section{REFERENCIAS}

BRANCO, A.L. Revoluções Industriais: Primeira, segunda e terceira revoluções. Disponível em: <http:// https://educacao.uol.com.br/disciplinas/geografia/revolucoes-industriais-primeirasegunda-e-terceira-revolucoes.htm> Acessado em: 27/07/2020.

EMILIANO1, J. R. L. Tese de Doutorado UNICAMP: Modelagem e Simulação de Redes loT, AdHoc e RFID. Novembro/2019.

EMILIANO2, J. R. L, et al. Internet of Things : An Overview of Architecture, Models, Technologies, Protocols and Applications. BTSYM 2018 SPRINGER. May/2019.

FIGUEIREDO Vinicius: Identificador visual automático para sistema de manufatura. : XX Encontro de Iniciação Cientifica e Tecnológica do IME, Rio de Janeiro, RJ,2019.

Henrique, J. B. R. Desenvolvimento de uma técnica de reconhecimento de padrões baseada em distância. Universidade Federal de Santa Catarina, 2003. Disponível em: <http://sharpbrains.com/pervasive-neurotechnology/>Acesso em: 24 jul. 2020.

IDA. The Internet of Things (IoT); International Digital Asset Management (IDA); July 2012. INTEL. Integrated Electronics Corporation. Big Data 101: Unstructured Data Analytics A Crash Course on the IT Landscape for Big Data and Emerging Technologies, June 2012. Disponível em: <https://www.intel.com/content/www/us/en/big-data/unstructured-data-analytics-paper.html> , acesso em 27/07/2020.

MANYIKA, J. e CHUI, M. e BROWN, B., et al. Big data: The next frontier for innovation, competition, and productivity. McKinsey Global Instituto, 2011.

SCHWAB, Klaus. A quarta revolução industrial. 1ạ edição, São Paulo: Edipro, 2019.

STEVAN, Junior. Indústria 4.0: Fundamentos, perspectivas e aplicações - São Paulo: Érica, 2018. 